\title{
Medizinprodukte: Keine UWG-Klage bei CE-Kennzeichnung
}

\author{
Eine OGH-Entscheidung stellt fest, dass sich Händler von Medizinprodukten auf die gesetzliche \\ Konformität von CE-gekennzeichneten Waren verlassen dürfen. Eine Klage wegen unlauterer \\ Verstöße gegen das Wettbewerbsrecht sei nicht zulässig.
}

Von Monika Ploier

Es ging um Schnelldiagnostika. Der OGH musste über einen Sachverhalt entscheiden, wonach das beklagte Medizinprodukteunternehmen In-vitro-Diagnosetests für Schwangerschafts- und Ovulationsprüfungen vertrieben hat, auf denen ein CE-Kennzeichen sowie die Nummer der Benannten Stelle angebracht waren. Die Klägerin warf der Beklagten unlautere Verstöße gegen das Medizinproduktegesetz MPG vor, da auf der Stückpackung und in der Gebrauchsanweisung bestimmte Informationen und Angaben fehlten. Am 22.09.2020 hat der OGH entschieden (4 Ob 135/20m) und erstmals bestätigt, dass der Inverkehrbringer bei Medizinprodukten auf ihre Rechtmäßigkeit vertrauen darf, wenn sie unter Einbindung einer Benannten Stelle zertifiziert wurden. Daher können auch keine Ansprüche aus dem UWG gegen den Händler oder Importeur geltend gemacht werden.

Grundsätzlich gilt für Medizinprodukte kein allgemeines Zulassungssystem durch eine bestimmte Behörde, sondern bringt vielmehr der Hersteller des Medizinprodukts selbst das CE-Kennzeichen an. Er erklärt damit, dass sein Medizinprodukt die gesetzlichen Vorgaben erfüllt. Nicht für alle Klassen von Medizinprodukten ist dabei die Einbindung einer Benannten Stelle erforderlich, sondern nur für Medizinprodukte, die nicht unter die Klasse I fallen. Trotz Einbindung der Benannten Stelle liegt die alleinige Verantwortung für die tatsächliche Konformität der Medizinprodukte mit den gesetzlichen Anforderungen beim Hersteller.

Entsprechend der europäischen und nationalen Rechtsgrundlagen gilt bei mit CE-Kennzeichen versehenen Medizinprodukten die Vermutung, dass diese gesetzlichen Anforderungen entsprechen, solange diese Konformitätsvermutung nicht widerlegt wurde.

Auf diese Grundlagen stützte sich das beklagte Medizinprodukteunternehmen. Es argumentierte zu dem Vorwurf, dass die von ihm vertriebenen Medizinprodukte nach einer umfassenden internen sowie externen Überprüfung durch eine unabhängige Benannte Stelle mit einem CE-Kennzeichen versehen worden waren. Und dabei gelte stets die Annahme, dass die entsprechenden Voraussetzungen für das Inverkehrbringen eingehalten seien. Denn sonst hätte die zuständige Behörde ein Überprüfungsverfahren einleiten müssen - was sie aber nicht getan hat.
Der OGH bestätigte diese Rechtsansicht und begründete dies damit, dass zu Recht eine Konformitätsvermutung für CE-gekennzeichnete Medizinprodukte bestehe. Der OGH erkennt zwar an, dass diese Vermutung widerlegbar ist, führt jedoch nicht näher aus, auf welche Art und Weise eine solche Widerlegung (zB auch im Rahmen eines UWG-Verfahrens) möglich ist. Vielmehr vertritt er die Ansicht, dass sich ein Medizinprodukteunternehmen, das CE-zertifizierte Medizinprodukte vertreibt, auf die zumindest „vertretbare Rechtsansicht“ iSd § 1 UWG stützen kann. Das bedeutet, dass nur dann eine nach dem UWG unlautere Geschäftspraktik vorliegt, wenn die generelle Norm nicht auch mit guten Gründen in einer Weise ausgelegt werden kann, dass sie Grund ging der OGH daher davon aus, dass das Medizinprodukteunternehmen aufgrund der Vermutung der aufrechten CEKennzeichnung davon ausgehen durfte, dass die vertriebenen Produkte den Anforderungen des MPG entsprechen und eine Überprüfung erfolgt ist. Diese Prüfung inkludiere auch Angaben, die auf der Kennzeichnung und in der Gebrauchsanweisung des Produkts anzubringen sind. Aus diesem Grund sah der OGH daher keinen Verstoß gegen $§ 1$ UWG.

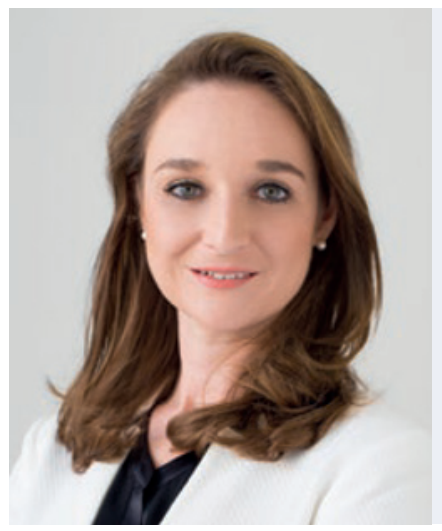

Dr. MONIKA PLOIER ist Anwältin bei HLMK Rechtsanwälte und auf Medizin- und Arbeitsrecht spezialisiert. Sie ist Verfasserin zahlreicher Publikationen und Lektorin für Medizin \& Recht an mehreren akademischen Bildungseinrichtungen. Monika Ploier ist Obfrau des Forschungsinstituts für Recht in der Medizin FIRM. 